\title{
SÍNTESE DE COMPLEXO FERRO(II)-NITROGÊNIO: ABORDAGEM DOS CONCEITOS DE REATIVIDADE E RETRO-DOAÇÃO PARA ALUNOS DE GRADUAÇÃO EM QUÍMICA
}

Fábio L. Pissetti, Rogério S. Nonô, Yoshitaka Gushikem e Gilson H. M. Dias*

Instituto de Química, Universidade Estadual de Campinas, CP 6154, 13084-971 Campinas - SP, Brasil

Recebido em 7/12/05; aceito em 28/7/06; publicado na web em 26/3/07

\begin{abstract}
SYNTHESIS OF THE IRON(II)-NITROGEN COMPLEX : CONCEPTS OF REACTIVITY AND BACK-BONDING FOR UNDERGRADUATE CHEMISTRY STUDENTS. There has been a considerable interest in coordination complexes of molecular nitrogen $\left(\mathrm{N}_{2}\right)$, partly due to a possible relationship between such complexes and the nitrogen activation process in nature. The present paper describes the synthesis and infrared spectroscopic characterization of an iron-nitrogen derivative with ethylenediamine-N,N,N',N'tetraacetate (edta) as an experiment for an undergraduate course. The topics covered here include synthesis, reactivity and spectroscopy.
\end{abstract}

Keywords: iron; edta; dinitrogen.

\section{INTRODUÇÃO}

O dinitrogênio é a molécula mais abundante na atmosfera terrestre e apesar disso não é aproveitada no metabolismo do nosso organismo. Contudo, a ativação do dinitrogênio atmosférico por sistemas biológicos é uma etapa fundamental no processo nutricional de muitos seres vivos e do nosso ecossistema. Enzimas em organismos vivos, como as da família da nitrogenase ${ }^{1}$ e complexos de alguns metais de transição ${ }^{2}$, apresentam as condições necessárias para ativar o dinitrogênio a temperatura e pressão amenas.

Tendo em vista o interesse por este fenômeno natural desde a caracterização do primeiro complexo com $\mathrm{N}_{2}$, pentamina (dinitrogênio)rutênio(II) $\left[\left(\mathrm{NH}_{3}\right)_{5} \mathrm{Ru}\left(\mathrm{N}_{2}\right)\right]^{2+}$ em $1965^{3,4}$, vários outros complexos contendo o nitrogênio molecular têm sido sintetizados e estudados devido, principalmente, à possibilidade de um melhor entendimento do mecanismo de fixação do dinitrogênio atmosférico realizado pela natureza. Este mecanismo é complexo e não completamente estabelecido, mas pode ser descrito de forma simplificada. Inicialmente ocorre a ligação do dinitrogênio ao metal no complexo/enzima com a conseqüente diminuição da força da ligação tripla entre os átomos de nitrogênio; usualmente, este processo é denominado de ativação do dinitrogênio. Em seguida procedemse simultaneamente a redução e protonação do nitrogênio, fornecendo amônia em temperatura e pressão baixas ${ }^{5}$. Atualmente, têmse estudado o mecanismo da ativação do dinitrogênio em comple$\operatorname{xos}^{6,7}$, tendo como objetivo desenvolver um catalisador mais efetivo com metais de transição, que promova a ruptura das ligações $\mathrm{N} \equiv \mathrm{N}$ em condições mais favoráveis, possibilitando um processo catalítico mais eficiente que o oneroso processo Haber-Bosch, usado ainda hoje na produção industrial da amônia e que utiliza pressão e temperatura altas ${ }^{8}$.

$\mathrm{O}$ dinitrogênio é relativamente inerte devido à sua ordem de ligação alta, a não polaridade da molécula e a uma alta energia de ionização. Além disso, apresenta também uma labilidade alta quando coordenado ao centro metálico, por causa do seu baixo caráter doador de densidade eletrônica pela ligação $\sigma$ e receptor de densidade eletrônica pela ligação $\pi$. Sabe-se que o grau de interação do nitrogênio na formação da ligação química é altamente dependente do centro metálico, do modo de ligação, assim como do ambiente reacional ${ }^{5,9}$.

*e-mail: dias@iqm.unicamp.br
A ativação da ligação tripla do dinitrogênio coordenado pelo modo terminal em complexo mononuclear acontece de duas formas diferentes, como mostrado na Figura 1. Quando a interação é fraca (estrutura A) somente se observa uma pequena retro-doação do metal para o $\mathrm{N}_{2}$, predominando o deslocamento da densidade eletrônica da ligação $\sigma$, enquanto que em uma interação forte (estrutura B) a retro-doação do metal para o $\mathrm{N}_{2}$ é dominante, então o deslocamento de densidade eletrônica para o orbital $\pi^{*}$ do dinitrogênio tem uma contribuição maior na coordenação do ligante 5 .

Ativação Fraca

Figura 1. Representação de alguns dos diferentes modos de coordenação do nitrogênio com metais em complexos. Reproduzido da ref. 5 com permissão da Elsevier

Uma ativação fraca praticamente não provoca mudança na ordem de ligação do $\mathrm{N}_{2}$, enquanto na ativação forte há uma diminuição da força de ligação, que passa a apresentar uma ordem de ligação menor que 3 (ligação tripla). Quando a molécula de nitrogênio passa a ter característica de ligação dupla (ordem 2) entre os átomos de nitrogênio, tanto a redução quanto a protonação são facilitadas, podendo assim promover a formação posterior de amônia em condições amenas ${ }^{5}$.

Além disso, a literatura descreve outros compostos mais complexos em que o dinitrogênio está coordenado ao metal. Por ex., as estruturas C e D na Figura 1 ilustram a ligação do dinitrogênio a dois metais, formando uma "ponte" entre os centros metálicos. Uma outra maneira de ligação do dinitrogênio descrita é a ligação lateral com o metal pela formação de duas ligações, como nas estruturas E, F e G. Em todos os casos, a ativação do nitrogênio depende das propriedades dos metais no complexo ${ }^{5}$.

Por essas razões, a preparação de um complexo ferro-nitrogênio é um experimento de ensino para alunos de graduação com um 
significante valor de aprendizado teórico e prático. Abordam-se conceitos de reatividade, labilidade e retro-doação. Além disso, o complexo é separado através de uma recristalização atípica, onde o produto final é solidificado a partir de sua forma oleosa.

Em acréscimo, os reagentes e materiais usados na experiência apresentam baixo custo e são de fácil obtenção, facilitando a preparação e implementação do experimento em um laboratório de ensino de graduação. $\mathrm{O}$ experimento, como descrito, foi realizado na disciplina de Química Inorgânica Experimental II (QI-542) ministrada para estudantes do penúltimo semestre do curso de Bacharelado/Tecnológica em Química da UNICAMP nos últimos 3 anos.

\section{PARTE EXPERIMENTAL}

O procedimento utilizado neste experimento teve como base o descrito por Basallote $e t a l^{10}$, que pode ser concluído em um período máximo de $16 \mathrm{~h}$. O Esquema 1 mostra um fluxograma indicando o tempo total utilizado nas aulas do curso onde o experimento foi realizado. Possivelmente, o experimento como descrito possa ser realizado em 3 aulas de $4 \mathrm{~h}$ cada. São necessárias quatro etapas para a preparação do produto final. Se no laboratório onde será feito o experimento tiver o ácido etilenodiaminatetrácetico, a primeira etapa não é necessária.

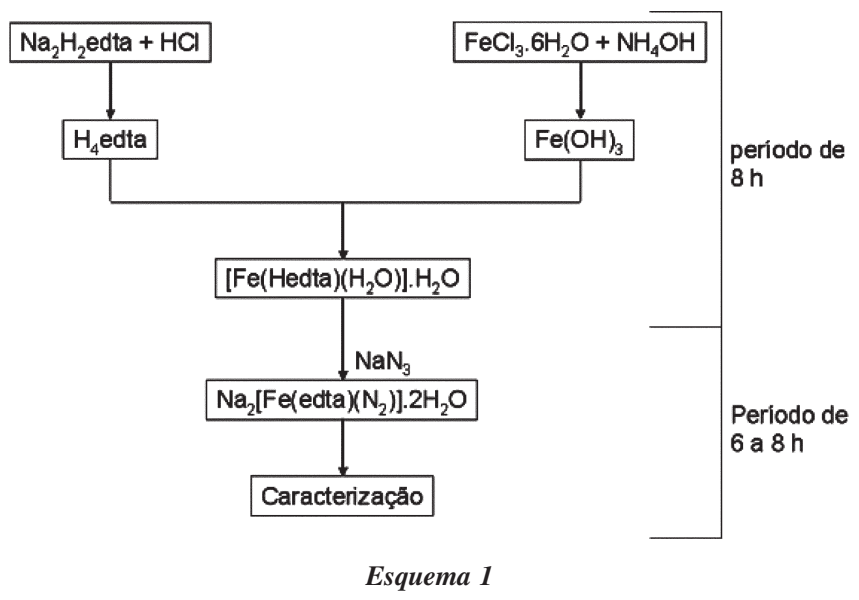

Preparação do ácido etilenodiaminatetrácetico ( $\mathbf{H}_{4}$ edta)

Dissolva 5,0 g (0,013 mol) de $\mathrm{Na}_{2} \mathrm{H}_{2}$ edta comercial em $300 \mathrm{~mL}$ de água destilada. Em seguida, adicione lentamente $\mathrm{HCl}$ concentrado sob agitação para que ocorra a precipitação do $\mathrm{H}_{4}$ edta. Evite um $\mathrm{pH}<3$ pelo controle da acidez com um papel indicador, porque o produto é solúvel em um meio muito ácido. Filtre o precipitado branco a vácuo e lave-o com porções de água até eliminar traços de acidez. Por fim, lave o precipitado com etanol e seque-o por 20 min em estufa a $100{ }^{\circ} \mathrm{C}$. Pese o produto e calcule o número de moles de $\mathrm{H}_{4}$ edta obtido, porque este deve estar em excesso de $20 \%$ em relação ao $\mathrm{Fe}(\mathrm{OH})_{3}$.

\section{Preparação do $\mathrm{Fe}(\mathrm{OH})_{3}$}

Dissolva um sal de $\mathrm{Fe}(\mathrm{III})$ (foi usado $\mathrm{FeCl}_{3} \cdot 6 \mathrm{H}_{2} \mathrm{O}$ ), em uma quantidade molar $20 \%$ menor que a obtida para $\mathrm{H}_{4}$ edta, em 100 $\mathrm{mL}$ de água destilada. Adicione lentamente hidróxido de amônio concentrado com agitação até que a solução apresente um pH básico. O produto é filtrado a vácuo e lavado sucessivamente com porções de água, até que a água de lavagem esteja neutra. Não é necessário secar completamente este produto vermelho escuro para seu uso na próxima etapa.

\section{Preparação do complexo precursor de Fe(III) - $\left[\mathrm{Fe}(\mathrm{Hedta})\left(\mathrm{H}_{2} \mathrm{O}\right)\right] . \mathrm{H}_{2} \mathrm{O}$}

Toda a quantidade de $\mathrm{H}_{4}$ edta e $\mathrm{Fe}(\mathrm{OH})_{3}$ preparada é colocada em um béquer de $250 \mathrm{~mL}$ com $100 \mathrm{~mL}$ de água destilada. Essa mistura é agitada por $2 \mathrm{~h}$ à temperatura de $70{ }^{\circ} \mathrm{C}$ em banho-maria. Depois desse período, a mistura reacional é resfriada e o $\mathrm{H}_{4}$ edta sólido em excesso, que não reagiu com o $\mathrm{Fe}(\mathrm{OH})_{3}$, é eliminado por filtração.

Diminua o volume total do filtrado até $50 \mathrm{~mL}$ com o auxílio de um evaporador rotativo, não deixando a temperatura do banho ultrapassar $70^{\circ} \mathrm{C}$. Transfira a solução resultante para um erlenmeyer. Adicione, com agitação, porções de acetona $(50$ a $100 \mathrm{~mL})$ para a precipitação do produto. Inicialmente, a solução é límpida, depois turva, para finalmente ocorrer a precipitação completa do produto amarelo-esverdeado. O produto é separado através de uma filtração a vácuo e seco à temperatura ambiente.

Neste primeiro período de $8 \mathrm{~h}$ de aula prática foi sintetizado o material de partida, restando apenas a última etapa de síntese e a caracterização do produto final.

\section{Preparação do complexo $\mathrm{Na}_{2}\left[\mathrm{Fe}(\right.$ edta $\left.)\left(\mathrm{N}_{2}\right)\right] \cdot 2 \mathrm{H}_{2} \mathrm{O}$}

Uma amostra de $0,5 \mathrm{~g}(0,0012 \mathrm{~mol})$ do complexo $\left[\mathrm{Fe}(\mathrm{Hedta})\left(\mathrm{H}_{2} \mathrm{O}\right)\right] \cdot \mathrm{H}_{2} \mathrm{O}$ é dissolvida em $20 \mathrm{~mL}$ de água previamente deaerada com fluxo de nitrogênio gasoso em um erlenmeyer de 125 mL. É aconselhável não adicionar um excesso de água, pois dificulta a precipitação do produto. Mantenha o fluxo do gás nitrogênio na solução e a agitação constante durante todo o procedimento. Em seguida, é adicionado a esta solução $0,5 \mathrm{~g}$ de azida de sódio $\left(\mathrm{NaN}_{3}-0,0077 \mathrm{~mol}\right)$. A solução imediatamente muda de amarela para laranja escura. Agite a solução reacional por $1 \mathrm{~h}$ e 30 min à $70{ }^{\circ} \mathrm{C}$ em banho-maria. Não ultrapasse o período de $2 \mathrm{~h}$ de reação, pois o produto com dinitrogênio coordenado lábil pode não ser obtido. A solução resultante é resfriada à temperatura ambiente, e adiciona-se 40-50 mL de etanol para se obter um óleo marrom, que fica aderido às paredes do recipiente. O sobrenadante é eliminado e adicionado mais $20 \mathrm{~mL}$ de etanol com agitação vigorosa. O produto aderido nas paredes do recipiente é raspado com uma espátula, resultando em um pó marrom. O etanol é descartado e o produto seco apenas com o fluxo de $\mathrm{N}_{2}$. O rendimento médio obtido pelos alunos foi de $70 \%$.

\section{Caracterização dos complexos}

Ambos os produtos obtidos com os ligantes $\mathrm{H}_{2} \mathrm{O}$ e $\mathrm{N}_{2}$ foram analisados através de espectroscopia vibracional na região de infravermelho em pastilhas de $\mathrm{KBr}$, usando um aparelho Nicolet Impact 410. O intuito desta comparação entre os produtos é evidenciar a ligação $\mathrm{Fe}-\mathrm{N}_{2}$. Estas medidas devem ser feitas logo depois da preparação do complexo ferro(II)-nitrogênio, porque a intensidade da banda referente ao estiramento do dinitrogênio diminui com o decorrer do tempo. Isto ocorre devido a labilidade do dinitrogênio coordenado, que vai sendo deslocado pela água presente na atmosfera. Além da diminuição da banda no espectro de infravermelho, se o produto for mantido em contato com o ar por um período mais prolongado vai deliquiescer, ficando com um aspecto oleoso.

\section{RESULTADOS E DISCUSSÃO}

O uso de edta em excesso de $20 \%$ na sua forma tetrácida $\left(\mathrm{H}_{4}\right.$ edta) em vez do edta dissódio $\left(\mathrm{Na}_{2} \mathrm{H}_{2}\right.$ edta) comercial é para que ocorra a formação única do complexo precursor $\left[\mathrm{Fe}(\mathrm{Hedta})\left(\mathrm{H}_{2} \mathrm{O}\right)\right] \cdot \mathrm{H}_{2} \mathrm{O}$ na reação do edta com $\mathrm{Fe}(\mathrm{OH})_{3}$, com um dos grupos carboxilatos do 
edta livre. Este reage prontamente com $\mathrm{N}_{3}^{-}$gerando o complexo de dinitrogênio pela substituição da água na sexta posição de coordenação do centro metálico. Por outro lado, o uso do $\mathrm{Na}_{2} \mathrm{H}_{2}$ edta levaria à formação de complexo com o edta hexacoordenado com o bloqueio de todas as coordenações do ferro, o que inviabilizaria a síntese posterior do complexo ferro-dinitrogênio.

A reação observada na síntese do complexo $\mathrm{Fe}-\mathrm{N}_{2}$ é análoga àquela que ocorre com a azida de sódio na bolsa de ar de veículos, onde o cátion $\mathrm{Na}$ é reduzido ao seu estado metálico, enquanto a azida é oxidada a dinitrogênio. Acredita-se que a reação de $\left[\mathrm{Fe}(\mathrm{Hedta})\left(\mathrm{H}_{2} \mathrm{O}\right)\right] \cdot \mathrm{H}_{2} \mathrm{O}$ seja iniciada pela adição do pseudo-haleto $\mathrm{N}_{3}{ }^{-}$ao centro metálico $\mathrm{Fe}(\mathrm{III})$, seguida de uma reação de oxi-redução:

$\left[\mathrm{Fe}(\mathrm{Hedta})\left(\mathrm{H}_{2} \mathrm{O}\right)\right] \cdot \mathrm{H}_{2} \mathrm{O}+2 \mathrm{NaN}_{3} \longrightarrow \mathrm{Na}_{2}\left[\mathrm{Fe}(\right.$ edta $\left.)\left(\mathrm{N}_{2}\right)\right] \cdot 2 \mathrm{H}_{2} \mathrm{O}+2 \mathrm{~N}_{2}$

$\mathrm{Na}$ reação acima a $\mathrm{H}_{2} \mathrm{O}$ é substituída pelo $\mathrm{N}_{3}$. $\mathrm{O}$ Fe(III) é reduzido a $\mathrm{Fe}(\mathrm{II})$, diamagnético, e oxida o íon $\mathrm{N}_{3}-\mathrm{N}_{2}$. Uma outra molécula de $\mathrm{N}_{3}^{-}$retira o $\mathrm{H}^{+}$do grupo carboxílico livre no edta coordenado.

No produto $\left[\mathrm{Fe}(\mathrm{II})(\mathrm{edta})\left(\mathrm{N}_{2}\right)\right]^{2-}$ (Figura 2), o dinitrogênio coordenado é lábil. Por esta razão é imprescindível manter a concentração do gás $\mathrm{N}_{2}$ alta pelo borbulhamento no meio durante toda a reação, para evitar que a $\mathrm{H}_{2} \mathrm{O}$ substitua $\mathrm{o} \mathrm{N}_{2}$ coordenado. Neste ponto do experimento, pode-se abordar o conceito de labilidade, comparando os estados de oxidação do ferro, a presença de ligantes diferentes e a necessidade do uso do fluxo de nitrogênio.

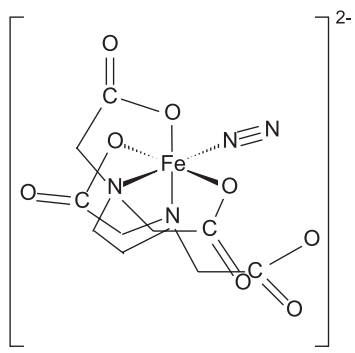

Figura 2. Estrutura sugerida para o complexo $\left[\mathrm{Fe}(\mathrm{edta}) \mathrm{N}_{2}\right]^{2-}$

$\mathrm{O}$ produto $\mathrm{Na}_{2}\left[\mathrm{Fe}\left(\right.\right.$ edta) $\left.\mathrm{N}_{2}\right] \cdot 2 \mathrm{H}_{2} \mathrm{O}$ é facilmente caracterizado por espectroscopia na região do infravermelho. A Figura 3 apresenta uma superposição dos espectros dos complexos de Fe(III) com a água e de Fe(II) com o dinitrogênio coordenado. A banda mais característica do complexo de $\mathrm{Fe}-\mathrm{N}_{2}$ corresponde ao modo de estiramento da ligação $\mathrm{N} \equiv \mathrm{N}$ do dinitrogênio ligado ao ferro. A posição desta banda é uma medida do caráter covalente da ligação metal-nitrogênio, a banda do dinitrogênio livre é observada em $2331 \mathrm{~cm}^{-1}{ }^{11}$. Uma diminuição do número de onda deste modo vibracional de estiramento sugere uma interação mais forte entre o $\mathrm{Fe}-\mathrm{N}_{2}$, evidenciando-se uma

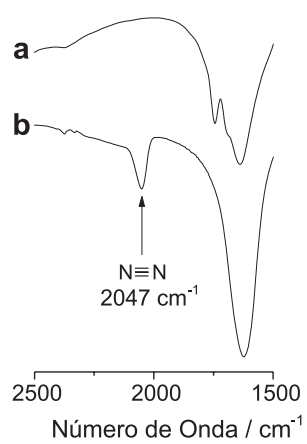

Figura 3. Espectro na região do infravermelho dos complexos: (a) $\left[\mathrm{Fe}(\mathrm{Hedta})\left(\mathrm{H}_{2} \mathrm{O}\right)\right] \mathrm{H}_{2} \mathrm{O}$ e (b) $\mathrm{Na}_{2}\left[\mathrm{Fe}(\mathrm{edta})\left(\mathrm{N}_{2}\right)\right] \cdot 2 \mathrm{H}_{2} \mathrm{O}$ polarização pequena na molécula de dinitrogênio ${ }^{5}$ por causa da retrodoação do $\mathrm{Fe}(\mathrm{II}) \mathrm{d}^{6}$, como ilustrado na Figura 4.

Os espectros no infravermelho mostrados na Figura 3 apresentam na região de $1620 \mathrm{~cm}^{-1}$ os estiramentos assimétrico e simétrico dos carboxilatos do edta. A banda que identifica o complexo Fe- $\mathrm{N}_{2}$ (Fe(II) $\mathrm{d}^{6}$ ) aparece por volta de $2047 \mathrm{~cm}^{-1}$ no espectro b, como descrito na literatura ${ }^{10,12}$. Neste ponto, uma comparação entre as energias da banda de estiramento do $\mathrm{N}_{2}$ em outros complexos de metal de transição permitiria verificar o grau de retro-doação em diferentes complexos. No complexo preparado há um sinergismo entre a ligação $\sigma$ e a retro-doação $\pi$, gerando um pequeno decréscimo no número de onda do estiramento do dinitrogênio. Isto acontece devido a um pequeno deslocamento de densidade eletrônica do metal para o orbital $\pi^{*}$ do dinitrogênio. Já o complexo $\left\{\left[\left(\mathrm{Me}_{3} \mathrm{SiNCH}_{2} \mathrm{CH}_{2}\right)_{3} \mathrm{~N}\right]\right.$ $\mathrm{Mo}_{2}\left(\mathrm{~N}_{2}\right) \mathrm{Mg}(\mathrm{THF})_{2}$ apresenta a banda de estiramento do dinitrogênio em $1719 \mathrm{~cm}^{-1}$, o que evidencia maior retro-doação. Isto ocorre porque o deslocamento de densidade eletrônica do metal para o orbital $\pi^{*}$ é mais intensa e, por consequiência a diminuição da ordem da ligação do dinitrogênio é maior. Outro complexo em que a retro-doação predomina fortemente é o $\left.\left\{\left[\mathrm{Pr}_{2} \mathrm{PCH}_{2} \mathrm{SiMe}_{2}\right)_{2} \mathrm{~N}\right] \mathrm{Zr}(\mathrm{OAr})\right\}_{2}\left(\mathrm{~m}-\mathrm{h}_{2}: \mathrm{h}_{2}-\mathrm{N}_{2}\right)$ com o estiramento do dinitrogênio em $751 \mathrm{~cm}^{-1}$. O número de onda da banda de estiramento da ligação $\mathrm{N} \equiv \mathrm{N}$ diminui com o acréscimo da densidade eletrônica no átomo central. Isto explica a razão da inexistência de complexos $\mathrm{Fe}-\mathrm{N}_{2}$ para $\mathrm{Fe}(\mathrm{III}) \mathrm{d}^{5}$, onde o sinergismo da retro-doação é quase nula ${ }^{13}$.

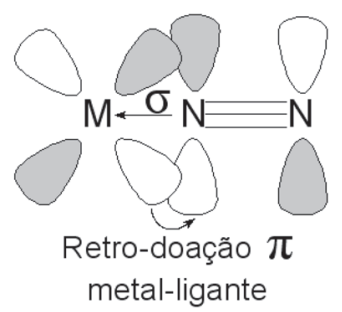

Figura 4. Esquema mostrando as interações $\sigma e \pi$ entre os orbitais $d$ do metal e do ligante. As setas indicam o deslocamento de densidade eletrônica, podendo-se verificar a transferência de densidade eletrônica do orbital d $\pi$ do metal para o orbital $p \pi *$ do ligante (retro-doação)

Além da espectroscopia na região do infravermelho, o complexo pode também ser caracterizado através de análise elementar e pelo espectro eletrônico na região ultravioleta/visível ${ }^{12}$. Com este dado pode-se estender o experimento e fazer uma introdução à teoria do campo cristalino ${ }^{14}$.

\section{CONCLUSÃO}

O método para a síntese do complexo ferro-dinitrogênio é relativamente simples e não há necessidade de nenhuma vidraria e de equipamentos diferenciados daqueles normalmente encontrados em um laboratório de ensino. Além disso, o experimento ilustra a obtenção não usual de um produto final, que na forma inicial de um óleo é posteriormente solidificado e seco apenas com um fluxo de nitrogênio.

A identificação do produto com o $\mathrm{N}_{2}$ coordenado ao átomo central de ferro é simples. Em princípio, é necessário apenas o espectro na região de infravermelho.

No decorrer do experimento alguns conceitos de química inorgânica, reatividade e retro-doação podem ser abordados utilizando-se aspectos práticos que surgiram na síntese dos complexos, auxiliando os alunos de graduação no aprofundamento dos conceitos abordados. 


\section{REFERÊNCIAS}

1. Yandulov, D. M.; Schrock, R. R.; Science 2003, 301, 76

2. Hidai, M.; Coord. Chem. Rev. 1999, 186, 99.

3. Allen, A. D.; Senoff, C. V.; J. Chem. Soc., Chem. Commun. 1965, 621.

4. Senoff, C. V.; J. Chem. Educ. 1990, 67, 368.

5. Fryzuk, M. D.; Johnson, S. A.; Coord. Chem. Rev. 2000, 200, 379.

6. Pool, J. A.; Lobkovsky, E.; Chirik, P. J.; Nature 2004, 427, 527.

7. MacLachlan, E. A.; Fryzuk, M. D.; Organometallics 2006, 25, 1550.

8. Shriver, D. F.; Atkins, P. W.; Langford, C. H.; Inorganic Chemistry, Oxford University Press: Oxford, 1990.
9. Tuczek, F.; Lehnert, N.; Angew. Chem., Int. Ed. 1998, 37, 2636.

10. Bassalote, M. G.; Lopez-Alcala, J. M.; Vizcaino, M. C. P.; GonzalezVilchez, F.; Inorg. Synth. 1986, 24, 207.

11. Nakamoto, K.; Infrared and Raman Spectra of Inorganic and Coordination Compounds, $5^{\text {th }}$ ed., John Wiley \& Sons: New York, 1997.

12. Lopez-Alcala, J. M.; Puerta, M. C.; Gonzalez-Vilchez, F.; Polyhedron 1984, 3,623 .

13. Chatt, J.; Dilworth, R.; Richards, R. L.; Chem. Rev. 1978, 78, 6.

14. Gushikem, Y.; Quim. Nova 2005, 28, 153. 